\title{
PROPOSTA DE PROTOCOLO PARA REABILITAÇÃO DE OSTEOARTROSE DE JOELHO EM CINESIOTERAPIA E HIDROTERAPIA PROTOCOLO DE REABILITAÇÃO PARA OSTEOARTROSE
}

\author{
PROTOCOL PROPOSAL TO REHABILITATION OF KNEE OSTEOARTHRITIS IN \\ KINESIOTHERAPY AND HYDROTHERAPY
}

Bruno Ruocco Verengue, Gustavo Calinin, Gustavo Ruas de Almeida, Juliana Leite Alves, Matheus Leite Praça, Nalim Raposo, Rosamaria Garcia Rodrigues

\begin{abstract}
RESUMO
Propor um protocolo de intervenção para osteoartrose de joelho utilizando exercícios em solo e em água. Revisão bibliográfica de artigos eletrônicos, nas bases PEDro, BVS e PubMed. Foram incluídos artigos publicados em revistas em português e inglês, entre 2009 e 2019. Foram encontrados 62 artigos, priorizando ensaios clínicos randomizados e que explorassem a temática proposta, foram excluídos artigos anteriores a 2009 e artigos de revisões bibliográficas, sendo selecionados 8 artigos de estudos originais randomizados para revisão. Verificou-se que intervenções em solo e em hidroterapia apresentaram resultados semelhantes em relação às variáveis estudadas: dor e funcionalidade. A única diferença notada foi o efeito da temperatura da água no relaxamento muscular e alívio da dor. Com isso, foi desenvolvido um protocolo para reabilitação de pacientes com OA de joelho, contemplando: aquecimento; fortalecimento e alongamento muscular; equilíbrio e propriocepção. A intervenção é composta por 12 semanas, 3 vezes por semana, sendo 2 vezes em solo e 1 vez na água. O protocolo conta com fortalecimento muscular dos principais grupos estabilizadores ativos do joelho. Ambos os métodos terapêuticos são efetivos nos quesitos abordados. Um programa de exercícios adequado pode evitar atrofia muscular e evitar limitações nas atividades diárias. As modalidades terapêuticas adequadas possibilitam controlar quadro álgico e a manutenção ou melhora da amplitude articular.
\end{abstract}

Palavras-chave: Osteoartrite. Exercício. Hidroterapia. Balneoterapia. Perna/joelho.

\begin{abstract}
To purpose a protocol of intervention to osteoarthritis knees using exercises in ground and in the water. Bibliography revision of electronic articles found on PEDro, BVC and PubMed. Articles published in portuguese and english were included, between 2009 and 2019. 62 articles were found emphasize random clinic rehearsal and so exploring the purposed theme. The results of 16 previous articles were excluded (before 2009 and bibliography revisions, 8 of them were chosen being original random to revision. After being analyzed, it was observed that ground intervention and hydrotherapy showed similar results in relation to the studied variables: pain and functionality, it was
\end{abstract}


noted and unique difference, the effect of water temperature in relaxed muscles and pain relief. Being so, it was developed a protocol to osteoarthritis knee rehabilitation patients, such as: heating, fortification and stretching, balance and proprioception. The intervention is made 12 weeks, 3 times a week, being so, in the ground twice and in the water once a week. The protocol is based on muscular fortification of the main active stabilizers of the knee. Both therapeutic methods are effective in the refuired items. An exercise well done program can avoid muscular atrophy and limitation of daily living activities. The therapeutic suitable modality enable to control pain and the maintenance or improvement of articular extent.

Keywords: Osteoarthritis. Exercise. Hydrotherapy. Balneotherapy. Lower leg/knee.

\section{INTRODUÇÃO}

A osteoartrose (OA) é uma doença reumática degenerativa que afeta as articulações sinoviais, alterando suas características, e dando origem a zonas de fibrilação e fissuração na cartilagem articular. Podem ser observadas microfraturas, cistos e esclerose no osso subcondral e formação de osteófitos nas bordas da articulação'.

O indivíduo pode sofrer influências externas ou internas ao longo da vida, que podem provocar o início precoce do processo degenerativo natural, como doenças inflamatórias ou infecciosas que afetam a estrutura cartilaginosa ou os traumas que envolvem a cartilagem, precipitando a osteoartrose. A evolução da degeneração articular, seja ela de causa idiopática ou secundária a trauma ou doença inflamatória, leva à desestruturação de todo o aparelho osteoligamentar e ao agravamento da deformidade $^{2}$. A influência genética é tida como um forte fator determinante da osteoartrose. Estudos epidemiológicos com gêmeos demonstram que a influência de fatores genéticos, visíveis radiograficamente, está entre 39 e 65\% na OA de joelho de mulheres ${ }^{3}$.

Cerca de 35\% dos casos de OA acontecem em indivíduos acima dos 30 anos de idade, e acometem preferencialmente os joelhos, e após os 50 anos, afeta quase todos os indivíduos, embora nem todos os acometidos tenham sintomas aparentes. Esse mal acomete tanto homens quanto mulheres e sua incidência aumenta com a idade e o peso ${ }^{4}$.

Com relação ao quadro clínico da artrose, seu início é progressivo. A dor, que é de origem mecânica, inicialmente associa-se à movimentação da articulação e, com a evolução da doença, com o repouso, aparecendo frequentemente à noite. Outros 
aspectos importantes são a rigidez, que normalmente é matinal, e a crepitação, que pode ocorrer com ou sem o movimento resistido. Além disso, na OA de joelho é comum o aparecimento de edema, frouxidão ligamentar, diminuição e/ou perda do movimento, contraturas capsulares, fraqueza muscular, espasmos, fibrose e deformidades progressivas em flexão, fatores estes agravantes para a instabilidade articular. Também pode haver deterioração da propriocepção ou senso de posição $\operatorname{articular}^{5}$.

O objetivo principal do tratamento da $O A$ de joelho não deve estar somente direcionado ao alívio dos sintomas e sim à melhora da capacidade funcional, isso porque não há recursos fisioterapêuticos que levem à cura da $O A$. A atuação da fisioterapia pode evitar que o impacto da osteoartrite cause maiores danos e leve à limitação de funcionalidade. Assim, deve ter como objetivos: a analgesia, a prevenção da perda e manutenção de trofismo e força muscular, e ganho de amplitude de movimento. Além disso, com a execução de um adequado programa fisioterapêutico outros benefícios podem ser alcançados, como a melhora da capacidade funcional e da qualidade de vida 6 .

A cinesioterapia é uma modalidade terapêutica definida como terapia através de movimentos corporais e exercícios físicos, englobando técnicas de alongamento muscular, fortalecimento, equilíbrio, propriocepção e capacidade aeróbica ${ }^{7,8}$.

O emprego da técnica visa melhorar a dor, força muscular, equilíbrio e mobilidade, consequentemente melhorar a capacidade funcional do indivíduo e os exercícios, quando bem executados e elaborados em um plano terapêutico, podem promover um possível efeito antinflamatório. Essa modalidade terapêutica pode ser trabalhada através de diversos modos, tais como: exercícios isométricos, isotônicos, resistidos ${ }^{9,10}$.

No emprego da técnica deve-se trabalhar alguns pontos essenciais, tais como consciência corporal, força muscular e alongamentos. O tratamento executado de forma correta e conjunta entre terapeuta e paciente pode gerar resultados mais satisfatórios, proporcionando maior qualidade de vida ${ }^{11}$.

A hidroterapia (do grego: "hydor", "hydatos" = água / "therapeia" = tratamento) é um dos recursos mais antigos da fisioterapia, sendo definida como o uso externo da água com propósitos terapêuticos ${ }^{12}$. 
Segundo Candeloro e Caromano ${ }^{13}$, este é um recurso fisioterapêutico que utiliza os efeitos físicos, fisiológicos e cinesiológicos, oriundo da imersão do corpo em piscina, como recurso auxiliar da reabilitação, ou na prevenção de patologias. Para melhoria e manutenção da amplitude de movimento (ADM) das articulações, do relaxamento e na diminuição da tensão muscular, as propriedades físicas da água atuam com um papel importante.

Dentre as diversas técnicas em que a hidroterapia se ramifica, uma das mais importantes e que mostra melhores resultados é a hidrocinesioterapia, que consiste em exercícios alternados no solo e na piscina terapêutica ${ }^{14}$. Os efeitos obtidos podem ser diminuição dos espasmos e quadro álgico, aumento da circulação sanguínea, melhora da ADM e fortalecimento muscular ${ }^{15}$.

Há métodos que podem ser utilizados na terapia, tais como o Watsu. Segundo Pereira ${ }^{16}$, trata-se de uma técnica baseada através de movimentos passivos juntamente com a sustentação na água e o movimento contínuo rítmico da água, com isso ocorre um relaxamento profundo. Além de auxiliar no quadro álgico e prevenção de outras patologias.

O Bad ragaz é uma técnica que associa a flutuação do paciente com auxílio de flutuadores no pescoço, braços, pelve e pernas e a realização de exercícios funcionais baseado na técnica de facilitação neuromuscular proprioceptiva (FNP) oriunda do Kabat ${ }^{17}$.

O Halliwick utiliza atividades com o objetivo de facilitar padrões de movimento variando sua dificuldade, com isso ela foi baseada nos princípios de hidrodinâmica e no desenvolvimento humano, fazendo com que o paciente se torne mais independente na água ${ }^{18}$.

Os princípios hídricos que estudam o comportamento dos líquidos em repouso e em movimento podem ser divididos em hidrostática e heterodinâmica. As principais propriedades físicas da água de maior alcance clínico sobre o corpo imerso são: densidade, empuxo ou flutuação, pressão hidrostática, turbulência e viscosidade ${ }^{19}$.

A densidade é a relação entre a massa e o volume do corpo imerso. Determina a capacidade que ele possui de flutuar ou submergir ao comparar a sua densidade com a da água ${ }^{20}$.

Empuxo ou flutuação, define-se por uma força de sentido contrário à gravidade, conferindo ao corpo imerso um efeito de sustentação e com isso o corpo flutua na 
superfície da água ${ }^{20}$. Os efeitos fisiológicos do empuxo ou flutuação são diminuição da pressão intrarticular, diminuição do espasmo muscular e tensão muscular.

A pressão da água exercida em várias direções sobre o corpo submerso é denominada pressão hidrostática (Lei de Pascal). Segundo Morris ${ }^{18}$, quanto maior a profundidade em que o corpo se encontra, maior será a pressão exercida sobre ele. Os efeitos fisiológicos da pressão hidrostática são analgesia, redução do edema, e ajuda na melhoria da capacidade respiratória.

A turbulência é o movimento que as moléculas de água fazem com um fluxo irregular sobre o corpo submerso, portanto quanto maior a velocidade do movimento, maior será a turbulência. Os efeitos fisiológicos da turbulência são diminuição da tensão muscular e alívio da dor ${ }^{13}$.

A viscosidade ou resistência do fluido é outra propriedade resultante da fricção entre as moléculas do fluido, que tendem a aderir-se à superfície do corpo que se move através dele, causando resistência ao seu movimento. Em uma comparação entre as piscinas frias e aquecidas, a viscosidade é menor em piscinas aquecidas, com isso quanto menor a temperatura, maior a viscosidade ${ }^{20}$.

Vistas as técnicas e modalidades terapêuticas que podem ser empregadas no tratamento da osteoartrose, o presente trabalho tem como objetivo elaborar um protocolo baseado em evidências para o tratamento da osteoartrose de joelho, propor um protocolo de intervenção fisioterapêutica para osteoartrose de joelho, utilizando a modalidade mais efetiva (cinesioterapia no solo e na hidroterapia) após a conclusão da análise.

\section{MÉTODOS}

Essa pesquisa trata-se de uma revisão bibliográfica, onde foram utilizados artigos eletrônicos, nas bases de dados PEDro, BVS e PubMed através dos descritores "osteoarthritis", "exercise", "hidrotherapy", "balneotherapy", "lower leg/knee", explorando a temática do uso da hidroterapia e da cinesioterapia para o tratamento da osteoartrose de joelho. A pesquisa foi realizada no período de janeiro a julho de 2019, sendo selecionados artigos publicados entre 2009 e 2019. Foram aplicados os critérios de inclusão: artigos publicados em revistas gratuitas nos idiomas 
português, inglês e espanhol, priorizando ensaios clínicos randomizados e que explorassem a temática proposta.

\section{RESULTADOS E DISCUSSÃO}

Foram encontrados 62 artigos, aplicados os critérios de inclusão, priorizando ensaios clínicos randomizados e que explorassem a temática proposta, resultaram 16 artigos, foram excluídos artigos anteriores a 2009 e artigos de revisões bibliográficas, sendo selecionados 8 artigos de estudos originais randomizados para revisão.

Wang et $\mathrm{al}^{21}$ compararam ao longo do tempo as mudanças em três grupos com sintomas iniciais avaliados pelo questionário Knee injury and Osteoarthritis Outcome - KOOS, (dor, rigidez, crepitação) e os efeitos secundários nas atividades de vida diária, esportes e recreação, o impacto da dor no joelho na qualidade de vida, ADM do joelho e teste de caminhada de seis minutos para investigar se exercícios aquáticos são superiores aos exercícios em solo na redução da dor. Os resultados demonstram que após 12 semanas de intervenção não houve diferença significativa na dor entre os grupos que realizaram exercícios na água e no solo $(p<0.820)$, porém os dois grupos apresentam melhora significante na qualidade de vida $(p<0,001)$ e na realização da atividade de vida diária $(p<0,001)$. Ambos modos de exercícios, água e solo, são efetivos para a melhora da qualidade de vida e funcionalidade em pacientes com OA de joelho, quando comparados ao grupo controle, e exercícios na água não são superiores a exercícios no solo para redução da dor. Os resultados da comparação entre as variáveis avaliadas estão descritos no Quadro 1.

Quadro 1 - Comparação entre as variáveis avaliadas em solo e na água Wang et $\mathrm{al}^{21}$

\begin{tabular}{|c|c|c|c|c|c|}
\hline $\begin{array}{c}\text { Wang } \\
2011\end{array}$ & Dor & Sintomas & Avd's & Esportes & QV \\
\hline $\begin{array}{c}\text { Água } \mathrm{x} \\
\text { controle 12 } \\
\text { sem }\end{array}$ & $<0,001$ & $<0,001$ & $<0,066$ & $<0,001$ & 0,001 \\
\hline $\begin{array}{c}\text { Água } \mathrm{x} \\
\text { controle 6 } \\
\text { sem }\end{array}$ & $<0,001$ & 0,024 & 0,356 & 0,062 & 0.074 \\
\hline
\end{tabular}




\begin{tabular}{|c|c|c|c|c|c|}
\hline $\begin{array}{c}\text { Solo x } \\
\text { controle 12 } \\
\text { sem }\end{array}$ & $<0,001$ & $<0,001$ & $<0,002$ & 0,001 & $<0,001$ \\
\hline $\begin{array}{c}\text { Água x } \\
\text { controle 6 } \\
\text { sem }\end{array}$ & 0,002 & 0,019 & 0,125 & 0,147 & 0,014 \\
\hline
\end{tabular}

Fisken et $\mathrm{al}^{22}$ compararam os efeitos de dois programas de exercícios aquáticos, sendo um realizado de forma sentada em meio aquático e acqua fitness (AF) realizados por 12 semanas, e ao final do programa avaliaram a qualidade de vida e capacidade funcional em idosos portadores de osteoartrose, através de escalas e testes funcionais. Posteriormente à realização dos programas de exercícios foi possível observar que Escores da Falls Efficacy Scale-International (FES-I) melhoraram significativamente no grupo AF $(p=0.03)$ comparado com o grupo controle. A análise indicou que ambos os grupos melhoraram significativamente 0400 $m$ walk test $(\mathrm{p}=0.04)$, e que o grupo AF melhorou significativamente o step test ( $\mathrm{p}=0.02)$ e a pontuação total da Arthritis Impact Measurement Scales 2 - AIMS2-SF $(\mathrm{p}=0.02)$. Não houve mudança significativa no timed up and go (TUG), sit to stand (STS) ou, handgrip strength (força de preensão palmar) observado para qualquer grupo ( $p>0.05$ ). O programa AF mostrou ser superior quanto ao número de benefícios que pode ser oferecido para idosos com osteoartrose, propiciando uma redução no medo de quedas e maior capacidade para executar as atividades de vida diária

Hale et $\mathrm{al}^{23}$ investigaram a eficácia de um programa de exercícios realizados na água visando especificamente o equilíbrio dos pacientes idosos para reduzir o risco de quedas, melhorar o equilíbrio e sua função física. Após a intervenção não houve diferença significativa nos grupos de intervenção e controle (Grupo intervenção: step test perna esquerda $p=0,000$; perna direita $p=0,004$. Grupo controle: step test perna esquerda $p=0,002$; perna direita $p=0,017$. Tempo de reação $p=0,03$. Contrast sensitivity $p=0,05)$. Diante dos resultados, o estudo demonstrou que exercícios na água não são efetivos para prevenção de quedas.

Taglietti et $\mathrm{al}^{24}$ compararam a eficácia de exercícios na água e terapia comportamental em paciente com $\mathrm{OA}$ de joelho. Ao longo do tratamento uma redução significativa do quadro álgico foi observada no grupo que realizavam exercícios na água. Diante dos resultados, o estudo demonstrou que exercícios na água quando 
comparado ao programa comportamental foram superiores em relação à dor $(p=0,021)$ e na função $(p=0,009)$ dos pacientes com OA de joelho.

Lim et $\mathrm{a}^{25}$ realizaram um programa de exercício aquático, e de exercício em solo para melhorar a função do joelho e reduzir a gordura corporal em pacientes com obesidade e osteoartrite do joelho. Embora não tenha sido encontrada diferença significativa nas características gerais entre os 3 grupos antes do exercício, a proporção de gordura corporal no grupo de exercícios em água diminuiu significativamente após a intervenção $(p=0,031)$. O grupo aquático apresentou melhora significativa na dor $(p<0,05)$, e qualidade de vida $(p<0,05)$. Notavelmente, $a$ redução da gordura corporal foi mais significante na redução da dor no grupo em água $(p=0,009)$ em relação ao grupo solo. Ambos os grupos de exercícios mostraram melhorias significativas na osteoartrite em comparação com o grupo controle. Foi constatado que os exercícios aquáticos pode ser uma ferramenta eficaz para pacientes com obesidade que têm dificuldades com o exercício físico devido à osteoartrite do joelho.

Stephen et $\mathrm{al}^{26}$ realizaram um estudo de ensaio clínico randomizado e controlado, tendo como finalidade nos trazer os benefícios dos exercícios físicos realizados tanto no solo, quanto no meio aquático em pacientes que serão submetidos a passar por um procedimento cirúrgico de artroplastia de joelho. Ao fim do tratamento foi observado que ambos métodos de tratamento não foram eficazes, tanto em quesitos funcionais $(p=0,739)$ quanto em relação de melhora do quadro álgico $(p=0,614)$, mas apenas os pacientes que foram submetidos ao tratamento no meio aquático relataram maior significância em questão da redução do quadro álgico imediatamente após o exercícios proposto $(p=0,019)$. Já na questão custo benefício o tratamento realizado no solo acaba sendo mais indicado.

O estudo de Waller ${ }^{27}$ et al teve como objetivo observar os efeitos do treinamento resistido aquático intensivo de 4 meses sobre a composição corporal e velocidade de caminhada em mulheres na pós-menopausa com OA, após a intervenção e após 12 meses de seguimento. Além da influência da atividade física no lazer. 87 mulheres divididas em 2 grupos (grupo de intervenção e grupo controle) o grupo de intervenção foram utilizados as seguintes escalas e testes: velocidade de caminhada em mais de $2 \mathrm{~km}$, a escala KOOS (Knee injury and Osteoarthritis Outcome Score) e a AFL (atividade física de lazer). Após a intervenção de 4 meses houve 
redução significativa de gordura corporal do grupo de intervenção aquática ( $\mathrm{p}=0.002$ e aumento na velocidade na marcha $(p=0.002)$. Não houve diferenças significativas no follow up de 12 meses para gordura corporal $(p=0.700)$ e velocidade da marcha $(p=0.032)$. Foi concluído que as intervenções do estudo foram efetivas para a diminuição de gordura corporal, bem como na velocidade de caminhada, o que auxilia em ganho de qualidade de vida e disfunções gerada pelas alterações da OA em mulheres na pós menopausa.

Dias et $\mathrm{a}^{28}$ realizaram um ensaio clínico randomizado para avaliar o efeito da hidroterapia em mulheres idosas com osteoartrose no joelho. Foi avaliada a dor, a funcionalidade e a função muscular. Uma amostra de 73 mulheres com 65 anos ou mais, distribuídas aleatoriamente em dois grupos: controle e hidroterapia. O grupo de hidroterapia recebeu um programa de intervenção em piscina aquecida, duas vezes por semana durante 6 semanas e um protocolo educacional, e o grupo controle recebeu apenas o protocolo educacional. Ao final das 6 semanas, o grupo de tratamento apresentou significantemente menos dor $(p=0,003)$ e maiores níveis de funcionalidade quando comparados ao grupo controle $(p=0,001)$. $A$ análise da função muscular também demonstrou melhores parâmetros no grupo de hidroterapia, a força aumentou significativamente em flexores de joelho $(p=0,040)$ e extensores $(p=0,050)$, a geração de força foi maior em flexores de joelho $(p=0,035)$ e a resistência foi maior para os extensores $(p=0,035)$.

Dias et $a^{28}$ apresentam que participantes de intervenções em água para $O A$ de joelho em um primeiro momento, tem significativamente menos dor no joelho e melhor funcionalidade quando comparados a pacientes que não realizaram nenhuma intervenção, e em segundo momento, intervenções na água aumentam significativamente a força muscular no joelho. Taglietti et al ${ }^{24}$ também concluem que pacientes submetidos a exercícios aquáticos obtém melhores resultados relacionados a melhora da dor, funcionalidade e qualidade de vida quando comparados a pacientes submetidos a apenas terapia comportamental. Os autores Wang et $\mathrm{a}^{21}$ concluem que tanto intervenções em água quanto em solo são efetivas para reduzir a dor e melhorar a qualidade de vida em pacientes com OA de joelho.

Embora os autores analisados obtiveram diferentes resultados após diferentes métodos de intervenção, ao final de todas as intervenções, quando são comparados exercícios tanto em água quanto em solo, com grupos de pacientes que não 
receberam nenhuma intervenção, todos os pacientes que realizaram os exercícios obtiveram melhora na dor, na funcionalidade ou na qualidade de vida. Portanto, independente da modalidade de exercício, água ou solo, sempre é preferível a realização de algum tratamento.

\section{PROTOCOLO DE EXERCÍCIOS}

Levando em consideração os resultados da revisão dos artigos selecionados, que concluiu que exercícios em solo e em hidroterapia são igualmente eficazes para melhora dos sintomas em pacientes com OA de joelho, foi proposto um protocolo abrangendo as duas modalidades de exercício para uma reabilitação mais efetiva e completa.

A intervenção tem duração de 12 semanas, com terapias três vezes na semana, sendo duas vezes por semana no solo e uma vez por semana na água, cada terapia tem duração de 45 minutos, e é estruturada em: aquecimento, fortalecimento muscular, treino de equilíbrio e propriocepção e alongamento muscular.

A sequência de exercícios de solo deve ser repetida nas duas sessões da semana, e a de hidroterapia apenas uma vez, cada terapia tem duração de 45 minutos.

Os exercícios em meio aquático podem ser realizados com adaptações, tal como: o uso de steps para pacientes que tenham baixa estatura.

Ao final de cada sessão o paciente deve ser orientado a realizar analgesia em domicílio, realizando aplicação de gelo no joelho por 30 minutos, duas vezes ao dia.

A descrição das intervenções em solo e em hidroterapia estão apresentadas nas tabelas 2 e 3, respectivamente. A progressão da carga seguiu o seguinte padrão: $1^{\text {a }}$ semana: sem carga; $2^{\underline{a}}$ e $3^{\underline{a}}$ semana: $0,5 \mathrm{~kg} ; 4^{\underline{a}}, 5^{\underline{a}}$ e $6^{\underline{a}}$ semana: $1 \mathrm{~kg} ; 7^{\underline{a}}, 8^{\underline{a}}$ e $9^{\underline{a}}$ semana: $1,5 \mathrm{~kg} ; 10^{\mathrm{a}}, 11^{\mathrm{a}}$ e $12^{\mathrm{a}}$ semana: $2 \mathrm{~kg}$.

Quadro 2 - Protocolo intervenção no solo

\begin{tabular}{|l|l|l|}
\hline $\mathbf{1}^{\mathbf{a}}$ semana & $\mathbf{2}^{\mathbf{a}}$ semana \\
$\begin{array}{l}\text { Aquecimento: marcha } \\
\text { frontal com dupla tarefa 2 } \\
\text { minutos }\end{array}$ & $\begin{array}{l}\text { Aquecimento: } \\
\text { marcha estacionária } \\
\text { cama elástica (2') }\end{array}$ & $\begin{array}{l}\text { Aquecimento: marcha } \\
\text { lateral com semi- } \\
\text { agachamento (2') }\end{array}$ \\
\hline
\end{tabular}


1- Isometria de extensão de joelho em sedestação. (4x15)

\section{2-Planti-flexão}

em sedestação. (2x10)

3- Dorsiflexão em sedesta ção. $(2 \times 10)$

4- OT apoiado em cadeira realiza abdução de quadril (2x10)

5- Em OT apoiado em cadeira realiza extensão de quadril $(2 \times 10)$

6- Subir e descer no step. (2x10)

7- Estratégia de tornozelo com oscilação ânteroposterior $3 \times 30 \mathrm{seg}$.

8- Em OT, com os pés juntos mantém a postura $2 \times 30$ seg.
Aquecimento: movimento de bicicleta em DD/SD (2')

1- Sentar e levantar da cadeira. (2x10).

2- Plantiflexão em sedest ação com caneleira de $1,5 \mathrm{~kg}(2 \times 15)$
1- Isometria de flexão de quadril com extensão joelho em DD. (5x15)

2-Planti-flexão em sedestação com caneleira

500gr. (2x10).

3 Dorsiflexão em sedesta ção com caneleira de $500 \mathrm{gr}$ em ante pé. (2x10)

4- OT apoiado em cadeira realiza abdução de quadril com caneleira de 500 gr $(2 \times 10)$

5- Em OT apoiado em cadeira realiza extensão de quadril com caneleira de 500 gr $(2 \times 10)$

\section{6- Subir e}

descer do step com caneleira de 500gr. (2x10)

7- Estratégia de tornozelo com oscilação ânteroposterior $3 \times 30$ seg.

8- Em OT em cima do colchonete, com os pés juntos e mantém (2x30seg).

\section{$5^{\text {a }}$ semana}

Aquecimento: dissociação

1- Flexão isotônica de quadril em DD com caneleira 500gr. (2x12).

2- Planti-flexão em sedestação com caneleira de $1 \mathrm{~kg}$. (2x12)

3- Dorsiflexão em sedesta ção com caneleira de 1 $\mathrm{kg} .(2 \times 12)$

4- Em OT apoiado em cadeira realiza abdução de quadril com caneleira de $1 \mathrm{~kg}(2 \times 10)$

5- Em OT apoiado em cadeira realiza extensão de quadril com caneleira de $500 \mathrm{gr}(2 \times 10)$

6- Subida e descida no step com caneleira de $1 \mathrm{~kg} \cdot(2 \times 12)$

7- Estratégia de tornozelo com oscilação laterolateral 3x30 seg.

8- Em apoio unipodal no solo. $(2 \times 20)$

\section{6a semana}

Aquecimento:

de cinturas em OT 1 minuto

1- Flexão isotônica de quadril em OT com caneleira de $0,5 \mathrm{~kg}$ alongamento ativo de quadríceps em OT $3 \times 30$ seg.

2- Em OT apoiado em 1- Flexão isotônica de quadril com caneleira de 1 $\mathrm{kg}(2 \times 12)$ 


\begin{tabular}{|c|c|c|}
\hline $\begin{array}{l}\text { 4- Em OT apoiado em } \\
\text { cadeira realiza abdução } \\
\text { de quadril com caneleira } \\
\text { de } 1,5 \mathrm{~kg}(2 \times 10) \\
5 \text { - Em OT apoiado em } \\
\text { cadeira realiza extensão } \\
\text { de quadril com caneleira } \\
\text { de } 1,5 \mathrm{~kg}(2 \times 10)\end{array}$ & $\begin{array}{l}\text { de quadril com caneleira } \\
\text { de } 2 \text { kg ( } 2 \times 10) \\
\text { 3- Em OT apoiado em } \\
\text { cadeira realiza extensão } \\
\text { de quadril com caneleira } \\
\text { de } 1 \mathrm{~kg}(2 \times 10) \\
\text { 4- Estratégia de } \\
\text { tornozelo em circundação } \\
\text { 3x30 seg. } \\
\text { 5- Plantiflexão em OT ( } 2 x \\
\text { 15) } \\
\text { 6- Dorsiflexão resistido } \\
\text { por faixa } \\
\text { elástica de resistência } \\
\text { média (2x12) } \\
\text { 7- Subir } \\
\text { descer do step associado } \\
\text { à elevação de membros } \\
\text { superiores (2x12) } \\
\text { 8- Em apoio unipodal em } \\
\text { superfície instável } 3 \times 20 \\
\text { segundos }\end{array}$ & $\begin{array}{l}\text { 2- Marcha lateral } \\
\text { faixa elástica de } \\
\text { resistência leve (2x12) } \\
\text { 3- Flexão de joelho em OT } \\
(2 \times 12) \\
\text { 4- Plantiflexão em OT } \\
\text { com caneleira de } \\
1 \mathrm{~kg}(2 \times 12) \\
\text { 5- Dorsiflexão resistido } \\
\text { por faixa elástica de } \\
\text { resistência forte (2x15) } \\
\text { 6- Subir } \\
\text { descer do step associado } \\
\text { à elevação de membros } \\
\text { superiores com halteres } \\
\text { de 0,5kg (2x10) } \\
\text { 7- Estratégia de tornozelo } \\
\text { em circundação } 3 \times 30 \\
\text { seg. } \\
\text { 8- Em apoio unipodal reali } \\
\text { za alcance com membro } \\
\text { superior em direção ao } \\
\text { solo (2x10) }\end{array}$ \\
\hline $\begin{array}{l}\text { Aquecimento: } \\
\text { alongamento } \\
\text { de isquiotibiais em SD } \\
\text { com faixa rígida } 3 \times 30 \\
\text { seg. } \\
\text { 1- Abdução de quadril } \\
\text { em SD com faixa elástica } \\
\text { resistência leve ( } 2 \times 10) \\
\text { 2- Pressionar bola entre } \\
\text { as pernas em SD (2x10) } \\
\text { 3- Flexão de joelho } \\
\text { em OT com caneleira de } \\
0,5 \mathrm{~kg}(2 \times 10)\end{array}$ & $\begin{array}{l}\text { 8ª semana } \\
\text { Aquecimento: circuito com } \\
\text { mudanças de direção } \\
\text { utilizando cones } \\
\text { minutos } \\
\text { 1- Flexão de joelho em OT } \\
\text { com caneleira de } 1 \mathrm{~kg} \\
\text { (2x10) } \\
\text { 2- Abdução de quadril } \\
\text { em sedestação com faixa } \\
\text { elástica resistência média } \\
\text { (2x12) } \\
\text { 3- Pressionar círculo magi } \\
\text { co entre os joelhos (2x12) }\end{array}$ & $\begin{array}{l}\text { 9a semana } \\
\text { Aquecimento: marcha } \\
\text { alterando sentidos ao } \\
\text { comando do terapeuta } 2 \\
\text { minutos } \\
\text { 1- Flexão de joelho em OT } \\
\text { com caneleira de 1,5kg } \\
2 \times 15 \\
2 \text { - Abdução de quadril } \\
\text { em sedestação com faixa } \\
\text { elástica } \\
\text { resistência forte (2x15) } \\
\text { 3- Adução de quadril com } \\
\text { paciente } \\
\text { em sedestação com faixa }\end{array}$ \\
\hline
\end{tabular}




\begin{tabular}{|c|c|c|}
\hline $\begin{array}{l}\text { 4- Plantiflexão em OT } \\
\text { com caneleira de } 1 \mathrm{~kg} \\
(2 \times 12) \\
5-: \text { semi-agachamento em } \\
\text { OT com bola suíça com } \\
\text { apoio do terapeuta (2x10) } \\
\text { 6- Extensão de quadril } \\
\text { com paciente } \\
\text { em OT apoiado na } \\
\text { cadeira com caneleira de } \\
2 \text { kg (2x10) } \\
\text { 7- Paciente em OT realiza } \\
\text { marcha em tandem } \\
\text { percorrendo distância de } \\
2 \text { metros } 4 x \\
\text { 8- Alongamento } \\
\text { de quadríceps em } \\
3 \times 30 \text { segundos }\end{array}$ & 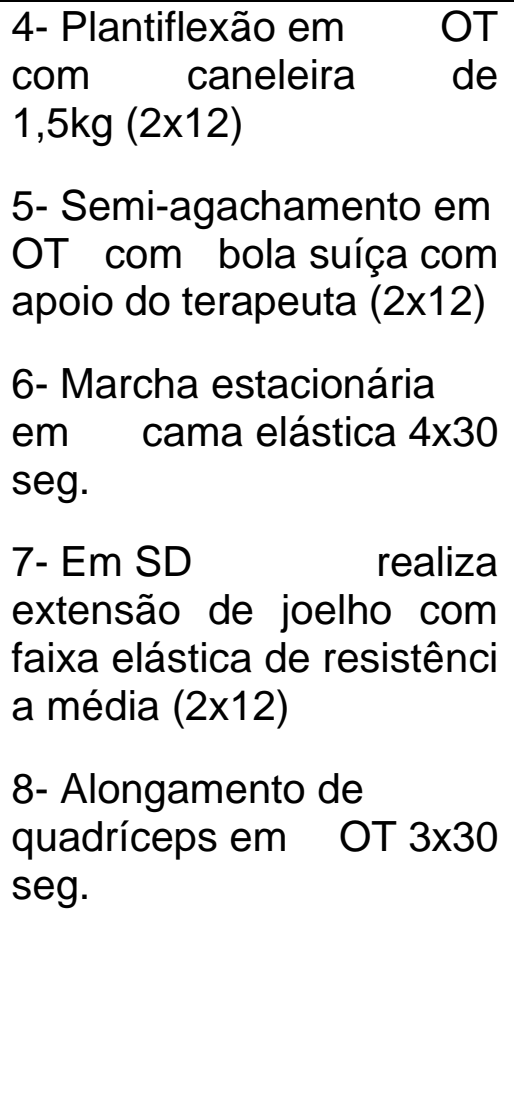 & $\begin{array}{l}\text { elástica resistência } \\
\text { média }(2 \times 12) \\
\text { 4- Agachamento em OT } \\
\text { com bola suíça com apoio } \\
\text { do terapeuta }(2 \times 10) \\
\text { 5- Marcha em calcâneo } \\
\text { percorrendo distância de } \\
2 \text { metros } 4 x \\
\text { 6- Marcha em plantiflexão } \\
\text { percorrendo distância de } \\
2 \text { metros } 4 \times \\
\text { 7- Marcha estacionária } \\
\text { em cama elástica com } \\
\text { caneleira de } 0,5 \mathrm{~kg} 4 \times 30 \\
\text { seg. } \\
\text { 8- Alongamento em SD } \\
\text { cruzando um Ml sob o } \\
\text { outro puxa bola suíça e } \\
\text { mantém } 3 \times 30 \text { seg. }\end{array}$ \\
\hline $\begin{array}{l}\text { 10a semana } \\
\text { Aquecimento: } \\
\text { mobilização patelar } \\
\text { 1- Flexão de joelho em OT } \\
\text { com caneleira de } 2 \text { kg } \\
2 \times 15 \\
2 \text { - Abdução de quadril } \\
\text { em OT com caneleira de } \\
0.5 \mathrm{~kg}(2 \times 15) \\
\text { 3- Adução de quadril } \\
\text { em SD com faixa elástica } \\
\text { resistência forte (2x12) } \\
\text { 4- Agachamento em OT } \\
\text { com bola suíça com apoio } \\
\text { do terapeuta ( } 2 \times 12) \\
5 \text { - Marcha em calcâneo } \\
\text { percorrendo distância de } \\
2 \text { metros com caneleira de } \\
0,5 k g 4 x\end{array}$ & $\begin{array}{l}\text { 11a semana } \\
\text { Aquecimento: } \\
\text { movimentos } \\
\text { de circundação com os } \\
\text { tornozelos em sentido } \\
\text { horário e anti-horário } \\
\text { 1- Flexão de joelho } \\
\text { em OT com caneleira de } 2 \\
\text { kg 2x15 } \\
\text { 2- Abdução de quadril } \\
\text { em OT com caneleira de } 2 \\
\text { kg (2x15) } \\
\text { 3- Adução de quadril com } \\
\text { paciente em OT com } \\
\text { caneleira de } 2 \text { kg (2x15) } \\
\text { 4- Extensão de quadril } \\
\text { com paciente em OT com } \\
\text { caneleira de } 2 \text { kg (2x15) }\end{array}$ & $\begin{array}{l}\text { 12-a semana } \\
\text { Aquecimento: alongament } \\
\text { o ativo de quadríceps em } \\
\text { OT 3x30 seg. } \\
\text { 1- Extensão de joelho em } \\
\text { isometria em SD com } \\
\text { caneleira de } 1 \mathrm{~kg} \text { (4x15) } \\
\text { 2- Flexão de joelho } \\
\text { em OT com caneleira de } 2 \\
\text { kg (3x15) } \\
\text { 3- Abdução de } \\
\text { em OT com caneleira de } 2 \\
\text { kg (3x15) } \\
\text { 4- Adução de quadril com } \\
\text { paciente em OT com } \\
\text { caneleira de } 2 \text { kg (3x15) } \\
\text { 5- Extensão de quadril } \\
\text { com paciente em OT com } \\
\text { caneleira de } 2 \text { kg (3x15) }\end{array}$ \\
\hline
\end{tabular}




\begin{tabular}{|c|c|c|}
\hline $\begin{array}{l}\text { 6- Marcha em plantiflexão } \\
\text { percorrendo distância de } \\
2 \text { metros com caneleira de } \\
0,5 \mathrm{~kg} \mathrm{4x} \\
\text { 7- Marcha estacionária } \\
\text { em cama elástica com } \\
\text { caneleira de } 1 \mathrm{~kg} 4 \times 30 \\
\text { seg. } \\
\text { 8- Alongamento de cadeia } \\
\text { posterior de membros } \\
\text { inferiores com faixa rígida } \\
\text { em SD } 3 \times 30 \text { segundos }\end{array}$ & $\begin{array}{l}\text { 5- Subir } \\
\text { descer do step com } \\
\text { caneleira de } 2 \mathrm{~kg}(2 \times 20) \\
\text { 6- Marcha em calcâneo } \\
\text { percorrendo distância de } \\
2 \text { metros com caneleira } \\
\text { de } 1 \mathrm{~kg} 4 \mathrm{x} \\
\text { 7- Marcha em plantiflexão } \\
\text { percorrendo distância de } \\
2 \text { metros com caneleira } \\
\text { de } 1 \text { kg } 4 x \\
\text { 8- Alongamento de } \\
\text { adutores de } \\
\text { em OT com quadril } \\
\text { apoio } 3 \times 30 \text { seg. }\end{array}$ & 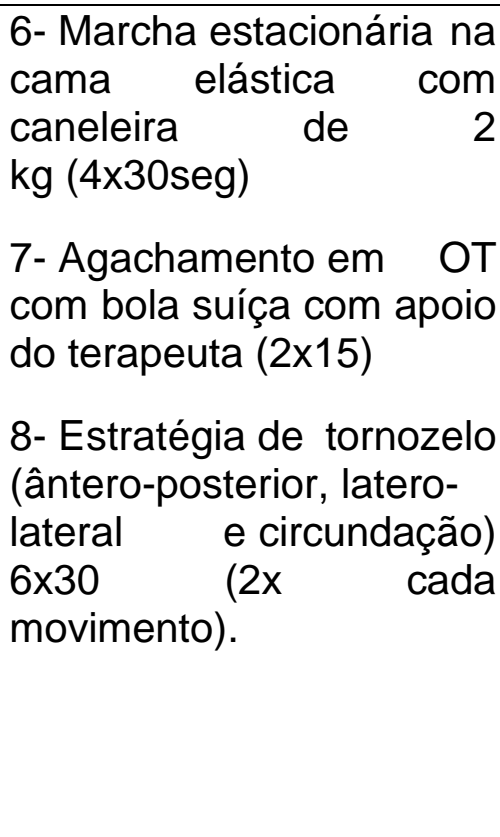 \\
\hline
\end{tabular}

Quadro 3 - Protocolo intervenção na água

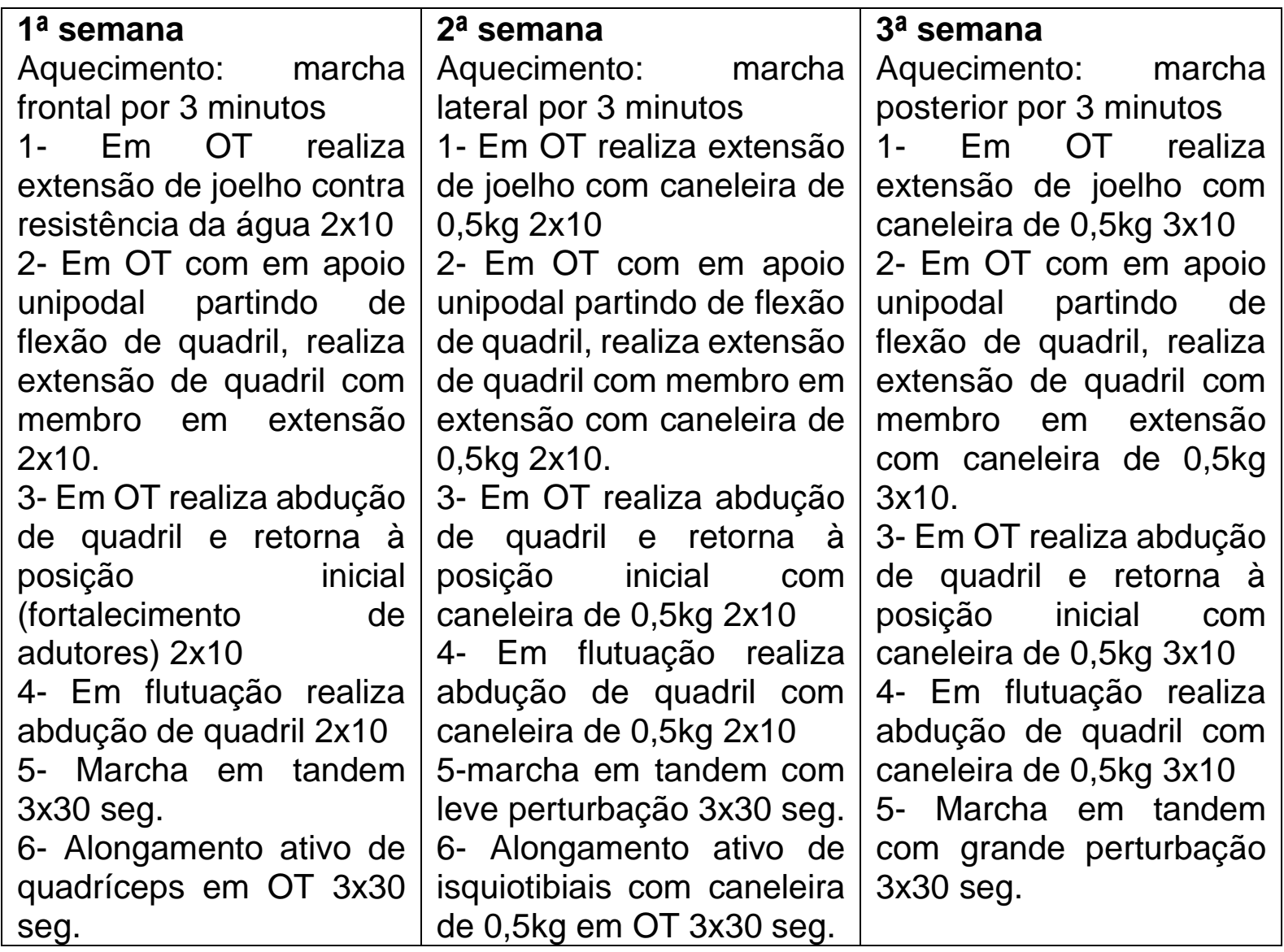




\begin{tabular}{|c|c|c|}
\hline $\begin{array}{lll}7-\quad \text { Em } & \text { OT } & \text { realiza } \\
\text { plantiflexão } 2 \times 10 & \\
8-\quad \text { Em } & \text { OT } & \text { realiza } \\
\text { dorsiflexão } & & \\
2 \times 10 & & \\
2 \times 10 & \end{array}$ & $\begin{array}{lrrr}7-\quad E m & \text { OT } & \text { realiza } \\
\text { plantiflexão com } & \text { caneleira } \\
\text { de } 0,5 \mathrm{~kg} 2 \times 10 & \\
\text { 8- } \quad \mathrm{Em} \text { OT } & \text { realiza } \\
\text { dorsiflexão com } & \text { caneleira } \\
\text { de } 0,5 \mathrm{~kg} & & \\
2 \times 10 & & \\
& \end{array}$ & $\begin{array}{l}\text { 6- Alongamento ativo de } \\
\text { adutores com caneleira } \\
\text { de } 0,5 \mathrm{~kg} \text { em OT } 3 \times 30 \text { seg. } \\
7-\quad \mathrm{Em} \mathrm{OT} \mathrm{realiza} \\
\text { plantiflexão com caneleira } \\
\text { de } 0,5 \mathrm{~kg} 2 \times 10 \\
8-\mathrm{Em} \text { OT realiza } \\
\text { dorsiflexão com caneleira } \\
\text { de } 0,5 \mathrm{~kg} \\
2 \times 10\end{array}$ \\
\hline 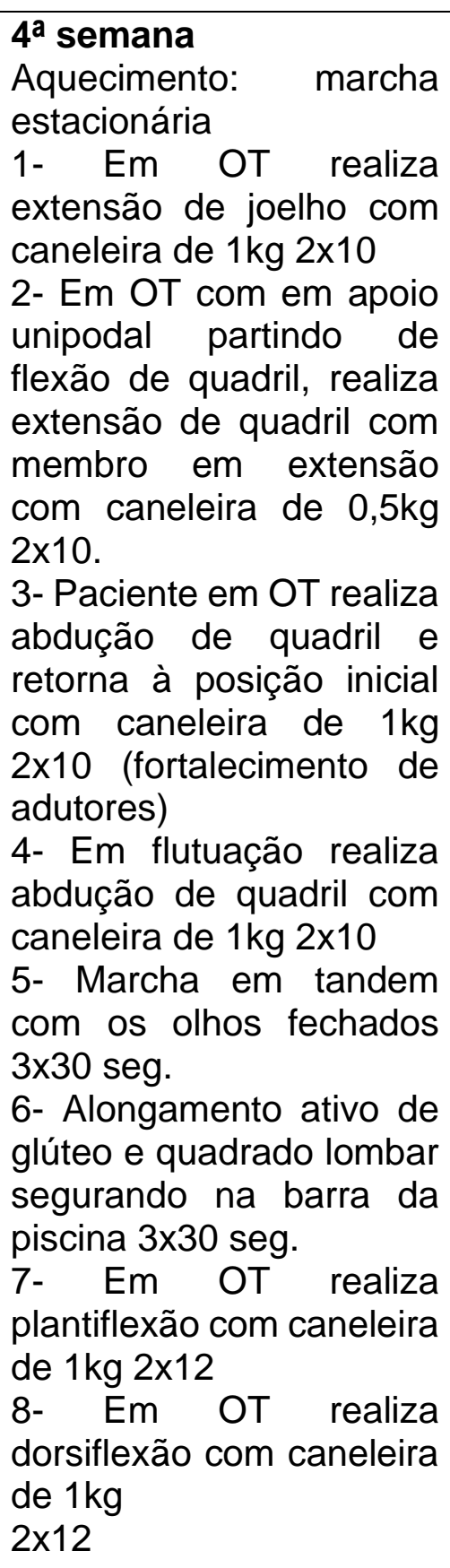 & 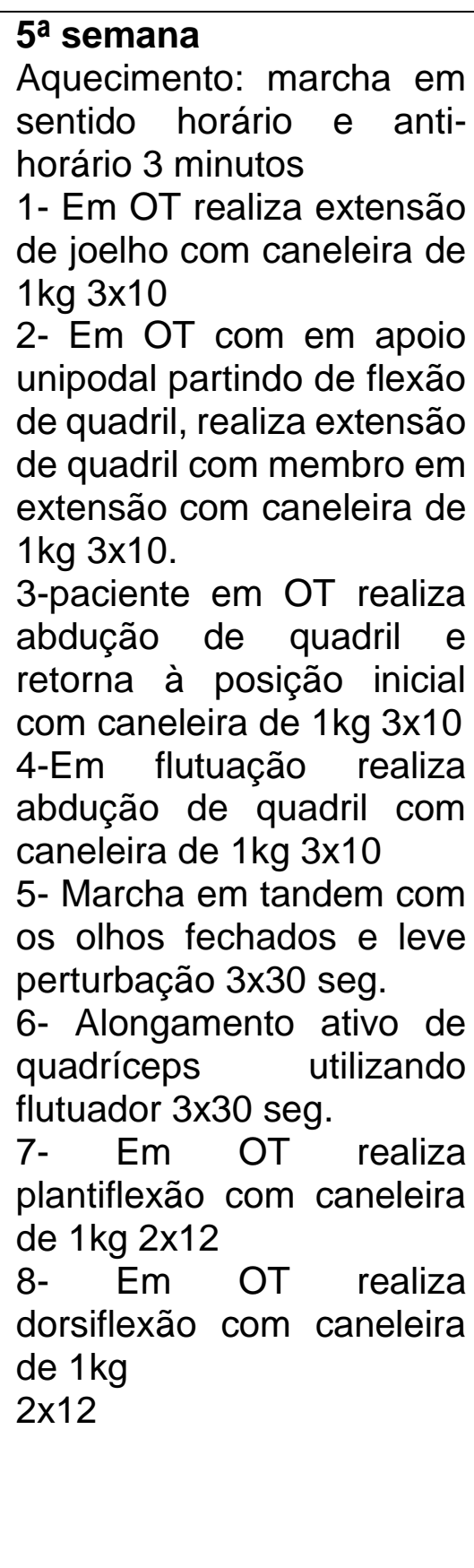 & 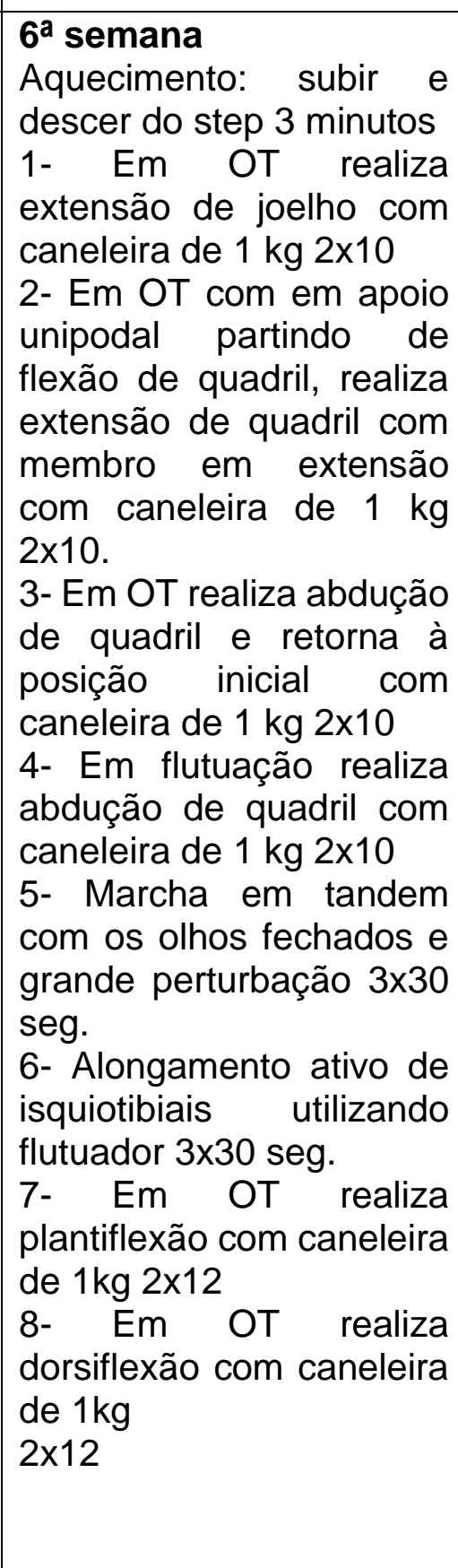 \\
\hline
\end{tabular}




\begin{tabular}{|c|c|c|}
\hline 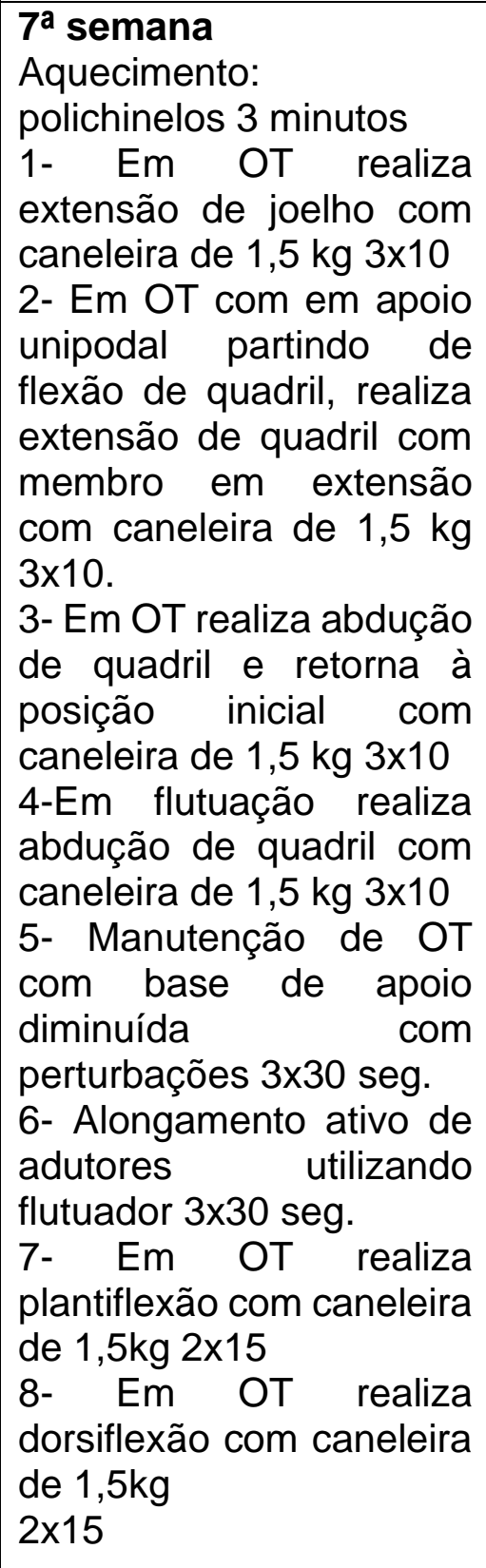 & 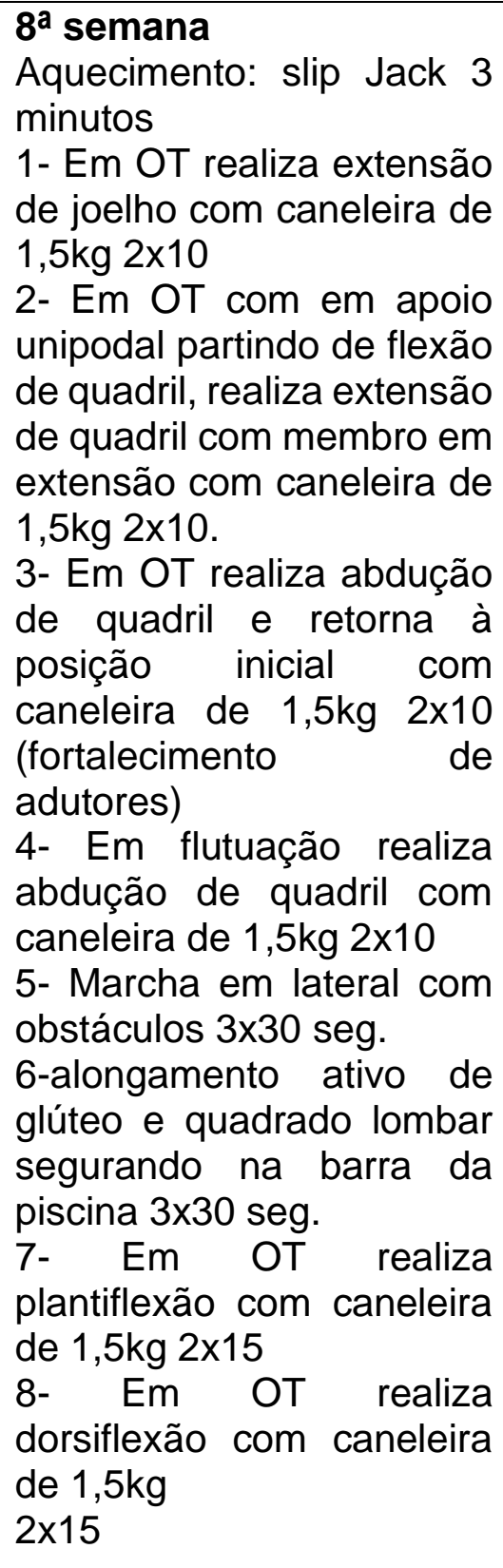 & 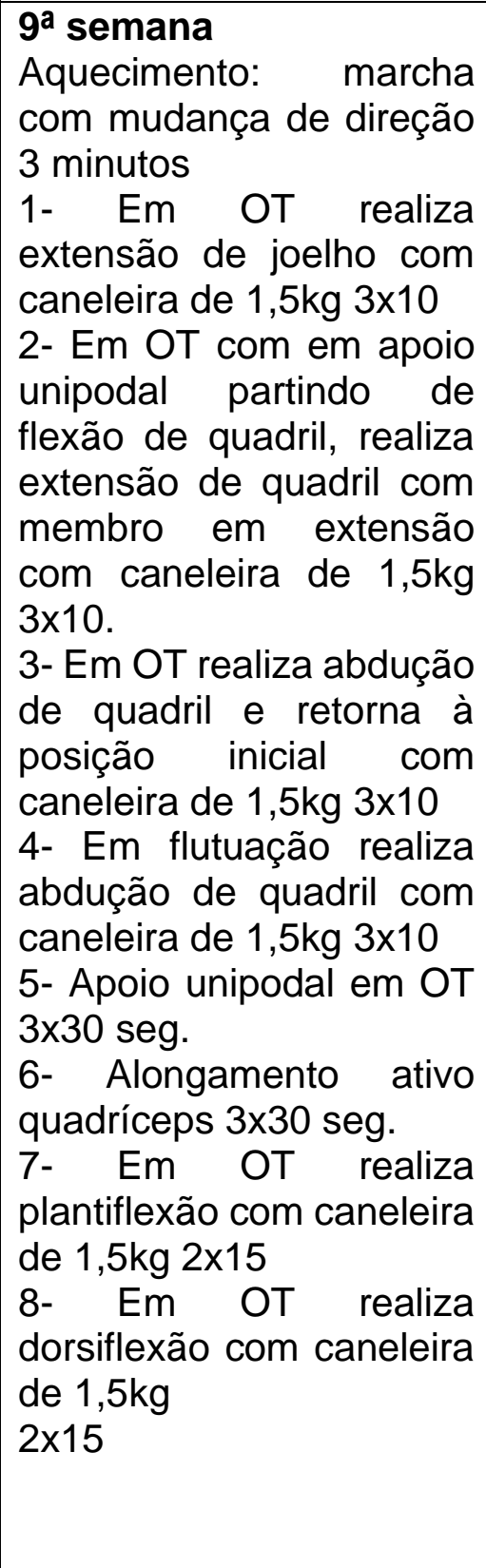 \\
\hline $\begin{array}{l}\text { 10ª semana } \\
\text { Aquecimento: subir e } \\
\text { descer do step elevando } \\
\text { MMSS } 3 \text { minutos } \\
\text { 1- Em OT realiza } \\
\text { extensão de joelho com } \\
\text { caneleira de } 2 \mathrm{~kg} 2 \times 10 \\
\text { 2- Em OT com em apoio } \\
\text { unipodal partindo de } \\
\text { flexão de quadril, realiza } \\
\text { extensão de quadril com } \\
\text { membro em extensão }\end{array}$ & $\begin{array}{l}11^{\text {a }} \text { semana } \\
\text { Aquecimento: marcha } \\
\text { posterior } 3 \text { minutos } \\
\text { 1- Em OT realiza extensão } \\
\text { de joelho com caneleira de } \\
2 \mathrm{~kg} 3 \times 10 \\
2-\text { Em OT com em apoio } \\
\text { unipodal partindo de flexão } \\
\text { de quadril, realiza extensão } \\
\text { de quadril com membro em } \\
\text { extensão com caneleira de } \\
2 \mathrm{~kg} 3 \times 10 \text {. }\end{array}$ & $\begin{array}{l}\text { 12a semana }^{\text {a }} \\
\text { Aquecimento: } \\
\text { polichinelos } 3 \text { minutos } \\
1-\text { Em OT realiza } \\
\text { extensão de joelho com } \\
\text { caneleira de } 2 \mathrm{~kg} 3 \times 15 \\
2-\text { Em OT com em apoio } \\
\text { unipodal partindo de } \\
\text { flexão de quadril, realiza } \\
\text { extensão de quadril com } \\
\text { membro em extensão }\end{array}$ \\
\hline
\end{tabular}




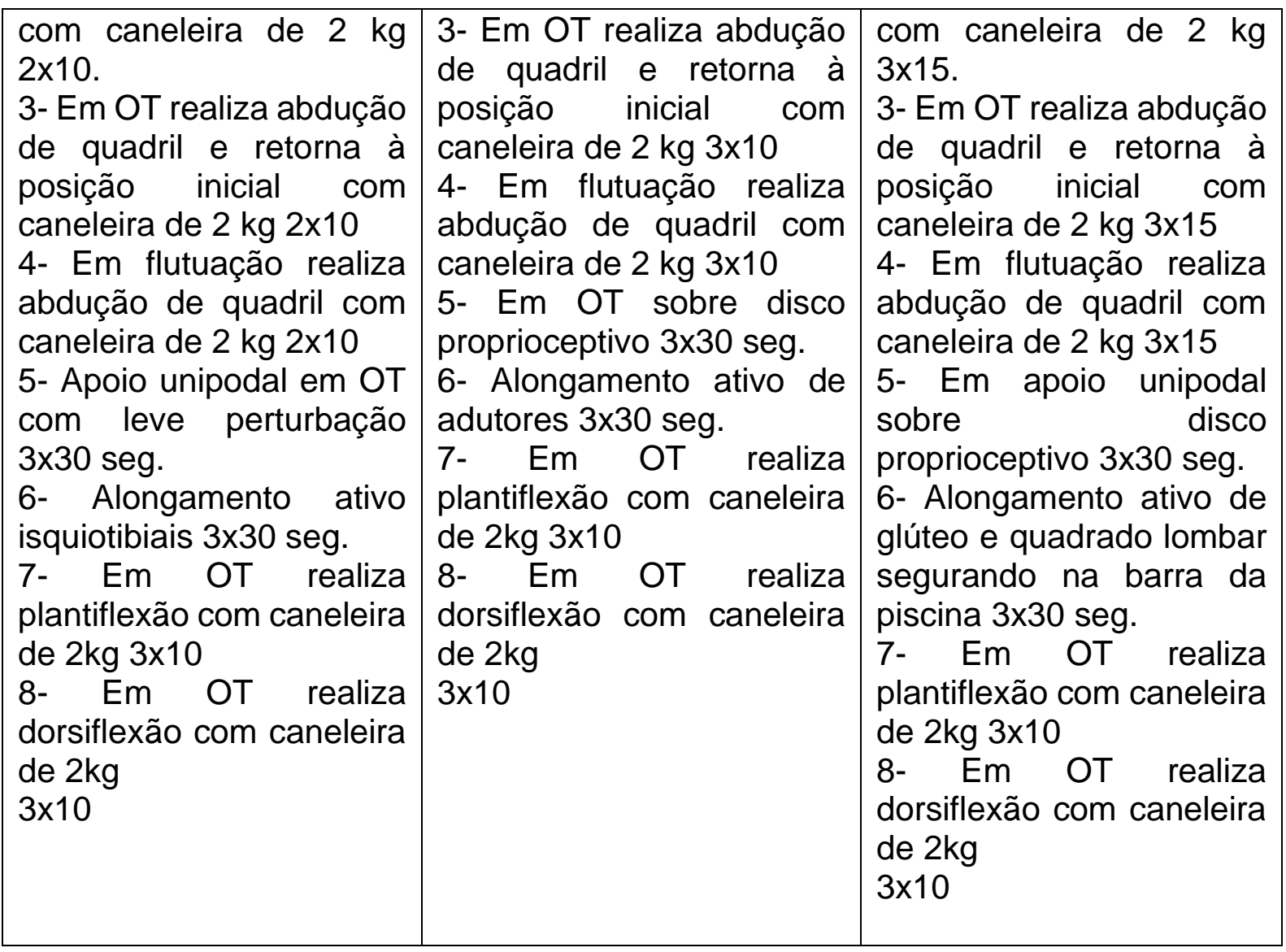

\section{CONCLUSÃO}

Foi observado que ambos os métodos terapêuticos são efetivos nos quesitos abordados (quadro álgico, fortalecimento muscular e funcionalidade).

Porem ao comparar o resultado dos artigos foi observado que houve uma redução no quadro álgico $\left(p=0,021^{24}\right)$ após sessões de hidroterapia em comparação às sessões realizadas em solo, enquanto nos outros aspectos não foram observadas diferenças significativas nos aspectos avaliados.

Um programa de exercícios bem formulado pode evitar atrofia muscular e, com isso, evitar limitações nas atividades de vida diária. As modalidades terapêuticas adequadas possibilitam controlar quadro álgico e a manutenção ou melhora da amplitude articular.

Em conclusão, os achados do presente estudo indicam que a osteoartrose de joelho acarreta prejuízos funcionais e dor incapacitante em pacientes portadores. Esses resultados demonstram a necessidade de uma intervenção efetiva considerando a funcionalidade e a força muscular para melhora da qualidade de vida 
e alívio do quadro álgico. Assim, devem-se desenvolver medidas terapêuticas efetivas no tratamento das alterações articulares. Sugere-se a realização de mais pesquisas na temática proposta e a aplicação do protocolo desenvolvido para avaliar a efetividade.

\section{REFERÊNCIAS BIBLIOGRÁFICAS}

1. MARQUES AP, KONDO A. A fisioterapia na osteoartrose: uma revisão de literatura. Revista Brasileira de Reumatologia. 1998; v.38, n. 2. Disponível em: <http://www.luzimarteixeira.com.br/wp-content/uploads/2009/09/osteartrose1.pdf>

2. CAMANHO GL. Tratamento da osteoartrose do joelho. Rev. Bras Ortop. 2001; v. 36, n. 5, p. 135-40. Disponível em: <http://www.luzimarteixeira.com.br/wpcontent/uploads/2009/09/osteoartrose-de-joelho>.pdf

3. SPECTOR TD, MACGREGOR AJ. Risk factors for osteoarthritis: genetics. OsteoArthritis and Cartilage. Twin Research \& Genetic Epidemiology Unit, St. Thomas' Hospital, London, UK. 2004; v.12. Disponível em: <https://www.sciencedirect.com/science/article/pii/S106345840300253X>

4. FRANCO LR, SIMÃO LS, PIRES EO, GUIMARÃES EA. Influência da idade e da obesidade no diagnóstico sugestivo de artrose de joelho. ConScientiae Saúde, Goiânia-GO. mar. 2009; n. 8, p. 41-46, 2. Disponível em: <https://www.redalyc.org/pdf/929/92911751006.pdf>

5. FACCI LM, MARQUETTI R, COELHO KC. Fisioterapia aquática no tratamento da osteoartrite de joelho: série de casos. Fisioterapia em Movimento, Curitiba, janeiro/março, 2007; v. 20, $\quad$ n. $1, \quad$ p. 17-27. Disponível em: <https://periodicos.pucpr.br/index.php/fisio/article/view/18829>

6. MINOR MA, HEWETT JE, WEBEL RR, ANDERSON SK, KAY DR. Efficacy of physical conditioning exercise in patients with rheumatoid arthritis and osteoarthritis. Arthritis and Rheumatism. Department of Medicine, University of Missouri, Columbia, 1989. Disponível em: < https://doi.org/10.1002/anr.1780321108>

7. KISNER C, COLBY LA. Exercícios Terapêuticos: Fundamentos e Técnicas. 3 ed. São Paulo: Manole, 1998. 
8. MASCARIN NC, VANCINI RL, ANDRADE ML, MAGALHÃES EP, LIRA CAB, COIMBRA IB. Effects of kinesiotherapy, ultrasound and electrotherapy in management of bilateral knee osteoarthritis: prospective clinical Trial. BMC Musculoskelet Disord. 2012; v.13: 182. Disponível em: <https://www.ncbi.nlm.nih.gov/pmc/articles/PMC3475115/>

9. OLIVEIRA NC, VATRI S, ALFIERI FM. Comparação dos efeitos de exercícios resistidos versus cinesioterapia na osteoartrite de joelho. Acta Fisiátr. 2016; 23(1):7-11. Disponível em: <http://www.actafisiatrica.org.br/detalhe_artigo.asp?id=610.>

10. JORGE MSG, ZANIN C, KNOB B, COMIN JDP, MOREIRA I, WIBELINGER LM. Efeitos da cinesioterapia na osteoartrite de joelho em idosos: revisão sistemática. Conscientiae saúde (Impr.); mar. 2018; 17(1): 93-100. Disponível em: <http://docs.bvsalud.org/biblioref/2018/10/916074/7428-49403-2-pb.pdf>

11. GAZZOLA JM, MUCHALE SM, PERRACINI MR, CORDEIRO RC, RAMOS LR. Caracterização funcional do equilíbrio de idosos em serviço de reabilitação gerontológica. Fisioterapia e Pesquisa, 2004; v.11, n.1, p.1-14. Disponível em: $<$ researchgate.net/profile/Renata_Cordeiro/publication/292407857_Caracterizacao_funcional _do_equilibrio_de_idosos_em_servico_de_reabilitacao_gerontologica/links/5bb7f5c8299bf10 49b700828/Caracterizacao-funcional-do-equilibrio-de-idosos-em-servico-de-reabilitacaogerontologica.pdf?origin=publication_detail>

12. CAROMANO FA. Princípios físicos que fundamentam a hidroterapia. Fisioterapia Brasil, 2019; v.3, p.394-402. Disponível em: <https://www.researchgate.net/profile/Fatima_Caromano/publication/238105255_PRINCIPIO S_FISICOS_QUE_FUNDAMENTAM_A_HIDROTERAPIA_PHYSICAL_PRINCIPLES_OF_H YDROTERAPY/links/5417990e0cf2218008bee957/PRINCIPIOS-FISICOS-QUE-

FUNDAMENTAM-A-HIDROTERAPIA-PHYSICAL-PRINCIPLES-OF-HYDROTERAPY.pdf>

13. CANDELORO JM, CAROMANO FA. Efeito de um programa de hidroterapia na flexibilidade e na força muscular de idosas. Rev. Bras. Fisioter, São Carlos, jul./ago. 2007; v.11, n.4, p.303-309, Disponível em: <http://www.scielo.br/pdf/rbfis/v11n4/a10v11n4.pdf> acesso em: 26 jan. 2019

14. MEEREIS ECW, FAVRETTO C, SOUZA J, GONÇALVES MP, MOTA CB. Influência da hidrocinesioterapia no equilíbrio postural de idosas institucionalizadas. Motriz, Rio Claro, abr./jun. 2013; v.19, n.2, p.269-277. Disponível em: <https://www.researchgate.net/profile/Carlos_Mota5/publication/262747084_Influence_of_hy drokinetic_therapy_in_postural_balance_of_institutionalized_elderly_people/links/0a85e53b2 
e8da6b67b000000/Influence-of-hydrokinetic-therapy-in-postural-balance-of-institutionalizedelderly-people.pdf> acesso em: 26 jan. 2019

15. SILVA KMOM, TUCANO SJP, KUMPEL C, CASTRO AAM, PORTO EF. Efeito da hidrocinesioterapia sobre qualidade de vida, capacidade funcional e qualidade do sono em pacientes com fibromialgia. Rev. Bras Reumatol, nov./dez. 2012; v.52, n.6, p. 846-857. Disponível em: <http://www.scielo.br/scielo.php?script=sci_arttext\&pid=S048250042012000600004>

16. PEREIRA SAP. A hidrocinesioterapia e sua influência na qualidade de vida de pacientes com fibromialgia. Fisioterapia Brasil. 2016; 15(1). Disponível em: <https://www.redalyc.org/pdf/929/92950553012.pdf>

17. RUOTI RG, MORRIS DM, COLE AJ. Reabilitação aquática. 2000; 1.ed. São Paulo: Manole.

18. MORRIS DM. Aquatic rehabilitation for the treatment of neurological disorders, Journal of Back and Musculoskeletal. Rehabilitation, 1994; 4:297-308. <Disponível em: https://content.iospress.com/articles/journal-of-back-and-musculoskeletal-rehabilitation/bmr44-09>

19. FINHOLDT MC. Análise da função autonômica sobre o sistema cardiovascular em humanos submetidos à mudança postural e imersão em água. Tese de Mestrado em Patologia Clínica. Universidade Federal do Triângulo Mineiro, UFTM, Brasil. 2007. Disponível em: <https://www.fontouraeditora.com.br/periodico/upload/artigo/517_1502367106.pdf>

20. CHÃO CC, IDE MR, FARIAS NC, ROSA AR, YNOUE AT. Fisioterapia aquática nas disfunções do aparelho locomotor. Anais do $2^{\circ}$ Congresso brasileiro de extensão universitária. set/2004. Universidade Estadual do Oeste do Paraná - UNIOESTE. Disponível em: <https://www.ufmg.br/congrext/Saude/Saude92.pdf>

21. WANG T-J, LEE SC, L SY, TUNG HH, WU S-FV, LIN YP. Comparing the efficacy of aquatic exercises and land-based exercises for patients with knee osteoarthritis. Journal of Clinical Nursing, 2010 nov; 20, 2609-2622. Disponível em: <https://www.ncbi.nlm.nih.gov/pubmed/21539629>

22. ALISON F, WATERS D, HING W, STEELE M, KEOGH J. Comparative Effects of 2 Aqua Exercise Programs on Physical Function, Balance, and Perceived Quality of Life in Older Adults with Osteoarthritis. Journal of Geriatric Physical Therapy, January 2015; 38(1):17-27. Disponível em: <https://www.ncbi.nlm.nih.gov/pubmed/24743752> 
23. HALE LA, WATERS D, HERBISON P. A Randomized Controlled Trial to Investigate the Effects of Water-Based Exercise to Improve Falls Risk and Physical Function in Older Adults with Lower-Extremity Osteoarthritis. Arch Phys Med Rehabil 2012; 93:27-34. Disponível em: <https://www.ncbi.nlm.nih.gov/pubmed/21982325>

24. TAGLIETTI M, FACCI LM, TRELHA CS, MELO FC, SILVA DW, SAWCZUK G, RUIVO TM, SOUZA TB, SFORZA C, CARDOSO JR. Effectiveness of aquatic exercises compared to patient-education on health status in individuals with knee osteoarthritis: a randomized controlled trial. Clinical Rehabilitation 2018; Vol. 32(6) 766 -776. Disponível em: <https://www.ncbi.nlm.nih.gov/pubmed/29417831>

25. LIM J-Y, TCHAI E, JANG S-N. Effectiveness of Aquatic Exercise for Obese Patients with Knee Osteoarthritis: A Randomized Controlled Trial. American Academy of Physical Medicine and Rehabilitation, August 2010; 1934-1482/10/\$36.00 Vol. 2, 723-731. Disponível em: <https://www.ncbi.nlm.nih.gov/pubmed/20709301>

26. Gill SD, McBurney H, Schulz DL. Land- based versus pool-based exercise for people awaiting joint replacement surgery of the hip or knee: results of a randomized controlled trial. Arch Phys Med Rehabil 2009; 90:388-94. Disponível em: <https://www.ncbi.nlm.nih.gov/pubmed/19254601>

27. WALLER B, OGONOWSKA-SLODOWNIK A, VITOR M, LAMBECK J, DALY D, KUJALA UM, HEINONEN A. Effect of Therapeutic Aquatic Exercise on Symptoms and Function Associated with Lower Limb Osteoarthritis: Systematic Review with Meta-Analysis. Phys Ther. 2014 Oct;94(10):1383-95. Disponível em: <https://www.ncbi.nlm.nih.gov/pubmed/24903110>

28. DIAS JM, CISNEROS L, DIAS R, FRITSCH C, GOMES W, PEREIRA L, SANTOS ML, FERREIRA PH. Hydrotherapy improves pain and function in older women with knee osteoarthritis: a randomized controlled trial. Brazilian Journal of Physical Therapy 2017; 21(6):449---456. Disponível em: <https://www.ncbi.nlm.nih.gov/pubmed/28733093> 